\title{
CARBAMAZEPINE AND THYROID HORMONES
}

Changes in serum thyroid hormone levels in 37 children with epilepsy receiving carbamazepine (CBZ) and valproic acid (VPA) were analyzed at the University of Chieti and University of Siena, Italy. Serum T4 and free thyroxine (FT4) levels were significantly lower in patients treated with CBZ and CBZ plus VPA than in controls, but were normal with VPA monotherapy. Serum T3 and FT3 levels were unaffected, and TSH levels were normal in all patients. Thyrotropin responses to thyrotropin-releasing hormone were similar in drug-treated and control groups, suggesting that hypothalamic function is unaffected. Serum thyroid hormone levels were not significantly correlated with serum AED concentrations. (Verrotti A, Basciani F, Morresi S et al. Thyroid hormones in epileptic children receiving carbamazepine and valproic acid. Pediatr Neurol July 2001;25:43-46). (Respond: Dr Alberto Verrotti, Department of Medicine, Section of Pediatrics, University of Chieti-Ospedale Policlinico, Via dei Vestini 5, 66100 Chieti, Italy).

COMMENT. As in previous studies in young adults, children with epilepsy treated with CBZ have decreased serum levels of T4 and FT4 and subclinical hypothyroidism, whereas T3, FT3, and TSH are unaffected. Valproate monotherapy causes no alteration in thyroid hormone levels. Previous studies in adults also showed that phenytoin causes decreased T4 and FT4 levels (see Progress in Pediatric Neurology I, PNB Publ, 1991;pp126-7). Thyroid supplements are usually not indicated in AED-induced reductions in T4 and FT4, unless signs of clinical hypothyroidism are present.

\section{HORMONE PROFILES WITH VALPROATE MONOTHERAPY}

Androgen, insulin, and lipid profiles were compared in young women and men treated with valproate (VPA) monotherapy and matched with controls taking lamotrigine (LTG), in a study at Western Infirmary, Glasgow, Scotland. Four obese VPA-treated women were hyperinsulinemic $(\mathrm{P}=0.05) ; 3$ had abnormal menstrual cycles and 1 a raised testosterone level. Obese patients of both sexes and VPAtreated men had elevated insulin levels. Testosterone and triglyceride levels were higher in VPA-treated women compared with those taking LTG. Only a minority of obese VPA-treated females had polycystic ovarian syndrome. (Stephen LJ, Kwan P, Shapiro D, Dominiczak M, Brodie MJ. Hormone profiles in young adults with epilepsy treated with sodium valproate or lamotrigine monotherapy. Epilepsia August 2001;42:1002-1006). (Reprints: Dr MJ Brodie, Epilepsy Unit, Department of Medicine and Therapeutics, Western Infirmary, Glasgow G11 6NT, Scotland).

COMMENT. VPA therapy may cause a subclinical hperinsulinemia in patients of either sex. In women, testosterone and triglycerides may also be elevated, indicative of polycystic ovarian syndrome. Women with obesity and a history of menstrual irregularities should receive a biochemical hormone screen. Those with abnormal chemistries should be advised to lose weight and avoid treatment with VPA.

\section{NEUROMUSCULAR DISORDERS}

\section{BELL'S PALSY AND REVIEW OF STEROID THERAPY}

The effect of corticosteroids in the treament of Bell's palsy in children $(<16$ years old) was evaluated by review of published trials and reported from the Hospital for Sick Children, Toronto, Canada. Of a total of 3392 reports identified, 8 
met inclusion criteria. Only one report involved children exclusively, with 21 treated and 21 controls (without placebo); the remainder included 1283 patients, mainly adults with few children who were not analysed separately. Only 5 of 89 trials were randomized (total 524 patients), and trial design, treatment schedule, and outcome measures were heterogeneous. Meta-analysis was not attempted. Four trials reported some benefit from corticosteroids, but the evidence is not conclusive. The routine use of corticosteroids in children with Bell's palsy is not recommended on the basis of this analysis and review. A definitive trial remains to be conducted. (Salman MS, MacGregor DL. Should children with Bell's palsy be treated with corticosteroids? A systematic review. I Child Neurol August 2001;16:565-568). (Respond: Dr Michael S Salman, Division of Neurology, Hospital for Sick Children, 555 University Ave, Toronto, ON M5G 1XS, Canada).

COMMENT. Present evidence based on review of published trials in children with Bell's palsy does not support the routine use of corticosteroids.

\section{$\underline{\text { TOXIC DISORDERS }}$}

\section{FACIAL SIGNS OF FETAL ALCOHOL SYNDROME}

Craniofacial measurements of 100 individuals exposed to alcohol before birth were compared to 31 controls in an anthropometric study to define fetal alcohol (FAS) or partial fetal alcohol syndrome (PFAS) at St Vincent Hospitals, Indianapolis. Six craniofacial measurements were identified that differentiated exposed vs nonexposed patients, with $96 \%$ accuracy, $98 \%$ sensitivity, and $90 \%$ specificity. A clinical diagnosis of FAS was made in 41 and PFAS in 59 children. Diagnostic measurements included 3 breadth (frontal, bigonial, and palpebral fissure), 2 circumference (head and maxillary arc), and 1 depth (midfacial). (Moore ES, Ward RE, Jamison PL et al. The subtle facial signs of prenatal exposure to alcohol: an anthropometric approach. L Pediatr August 2001;139:215-219). (Reprints: Elizabeth S Moore PhD, Quality Management, St Vincent Hospitals and Health Serrvices, 2001 West 86th St, PO Box 40970, Indianapolis, IN 46240).

COMMENT. Signs of definite FAS are prenatal and postnatal growth deficiency and brain and craniofacial abnormalities. Short palpebral fissures, smooth philtrum, thin upper lip, and midfacial hypoplasia are the most common facial anomalies. The present study of subtle facial signs of PFAS and diagnostic anthropometric measurements will permit the recognition of a wider range of children with alcohol-related birth defects and lead to counseling and prevention of further cases in the family.

\section{ATTENTION DEFICIT DISORDERS}

\section{DIETARY SUPPLEMENTS FOR ADHD: A CONTROLLED TRIAL}

The effect of docosahexaenoic acid (DHA) supplementation $(345 \mathrm{mg} / \mathrm{d}$ ) on the symptoms of attention deficit/hyperactivity disorder (ADHD) was determined in 63 children, ages 6 to 12 years, at the Mayo Clinic and Baylor College of Medicine, Houston, TX. All were receiving effective therapy with stimulant medication, and were assigned at random, double-blind, to DHA or placebo groups for 4 months. Outcome was determined by scores on laboratory measures of inattention and impulsivity (TOVA, Color Trails), performed after discontinuing medication for 24 hours, and scores on parent rating scales (Child Behavior Checklist, Conners' Rating Scale), completed while continuing medication. Plasma 